\title{
Central-force model with the next-nearest-neighbor interaction: A series approach
}

\author{
Jian Wang \\ Department of Physics, The University of Hong Kong, Pokfulam Road, Hong Kong
}

(Received 20 April 1995)

\begin{abstract}
A central-force model with the next-nearest-neighbor interaction is studied using a series-expansion technique on a honeycomb lattice. The bulk modulus exponent is calculated and found to be $f_{B}=3.96 \pm 0.01$. This value is very close to the bulk modulus exponent for the bond-bending model $f_{B}=3.96 \pm 0.04$, calculated by Zabolitzky and co-workers using a transfer-matrix technique on the same lattice. Our calculation suggests that these two models are in the same universality class.
\end{abstract}

\section{INTRODUCTION}

Recently much attention ${ }^{1-6}$ has been directed towards randomly diluted elastic networks. Various models have been studied, such as the central-force model, ${ }^{1,3,5}$ the bondbending model, ${ }^{2,4}$ the granular disk model, ${ }^{7,8}$ etc. The bondbending model is perhaps the one we understand the best. For this model the bulk modulus $B$ goes to percolation threshold. The bulk modulus exponent $f_{B}$ can be defined by $B \sim\left|p-p_{c}\right|^{f_{B}}$. Based on numerical result and scaling argument it has been suggested that ${ }^{4,9-11}$ for the bond-bending model, one has the relation

$$
f_{B}=t+2 \nu,
$$

where $\nu$ is the correlation length exponent for percolation and $t=(d-2) \nu+\zeta_{\mathrm{Re}}$ (Ref. 12) is the exponent for the conductivity of the analogous randomly diluted resistor network defined by $\Sigma(p) \sim \sigma\left|p-p_{c}\right|^{t}$, where $\sigma$ is the conductance of an occupied bond (which occurs with probability $p$ ) and the vacant bonds occurring with probability $1-p$ have zero conductance. Here $\zeta_{\mathrm{Re}}$ is the conductivity crossover exponent for the resistor network. In terms of this crossover exponent, we have $f_{B}=d \nu+\zeta_{\mathrm{Re}}$. Zabolitzky et al. ${ }^{13}$ have calculated $f_{B}$ for the bond-bending model on a honeycomb lattice by computer simulation using a transfer-matrix method. They found that $f_{B}=3.96 \pm 0.04$. Using $t=1.30$ (Ref. 14) and $\nu=4 / 3$, we see that Eq. (1) is almost exact. Recently, further efforts have been made to understand the physics behind Eq. (1). For instance, it has been proved ${ }^{15}$ that the elastic splay crossover exponent ${ }^{5} \zeta_{\mathrm{SP}}$ of the bond-bending model, which is related to the bulk modulus exponent $f_{B}=d \nu+\zeta_{\mathrm{SP}},{ }^{11,8,15}$ is the same as the conductivity crossover exponent $\zeta_{\mathrm{Re}}$ for the lattice animal. Using the series-expansion method the elastic splay crossover exponent $\zeta_{\text {SP }}$ has been calculated ${ }^{16}$ on a honeycomb lattice up to 13th order. Most recently, we ${ }^{17}$ have extended the series to 18th order which enables us to estimate the exponent very accurately. We found that $\zeta_{\mathrm{SP}}=1.31 \pm 0.02$, which is in very good agreement with $\zeta_{\mathrm{Re}}$.

In this paper, we consider the randomly diluted centralforce model with the next-nearest-neighbor (NNN) interaction in two dimensions whose Hamiltonian can be written as

$$
H=H_{\text {cen }}+H_{\mathrm{NNN}},
$$

where

$$
H_{\mathrm{cen}}=\frac{1}{2} \sum_{i, j} k_{1}\left[\left(\vec{u}_{i}-\vec{u}_{j}\right) \cdot \vec{R}_{i, j}\right]^{2}
$$

and

$$
H_{\mathrm{NNN}}=\frac{1}{2} \sum_{\mathrm{NNN}(i, j)} k_{2}\left[\left(\vec{u}_{i}-\vec{u}_{j}\right) \cdot \vec{R}_{i, j}\right]^{2} .
$$

Here $k_{1}$ and $k_{2}$ are the elastic coupling constants of the nearest-neighbor sites and the next-nearest-neighbor sites, respectively, $\vec{u}_{i}$ is the displacement of the site $i$, and $\vec{R}_{i, j}$ is the unit vector along sites $i$ and $j$. The summation in Eq. (3) is over the nearest-neighbor sites, whereas in Eq. (4) it is over the next-nearest-neighbor sites.

This model has been considered by Garboczi and Thorpe, ${ }^{18}$ but with two independent concentrations $p_{1}$ and $p_{2}$, for Eqs. (3) and (4), respectively. Their study was restricted to the effective medium theory. In this paper, we consider the following random dilution: bonds are present with probability $p$, absent with probability $1-p$. For a given cluster, Eq. (3) is present for every bond in the cluster, Eq. (4) is present for sites $i$ and $j$ provided that they are the NNN sites. When $k_{2}$ in Eq. (4) is zero, we obtain the central-force model. At present there is disagreement in the literature as to whether the central force model and the bond-bending model are in the same universality class. Although most calculations ${ }^{1,3,16}$ suggest that they are not in the same universality class, a recent one ${ }^{6}$ suggests that they are. The disagreement may be due to anomalous corrections to scaling, the treatment of which, in turn influences the exact location of the critical threshold, and thereby the determination of the critical exponents. In our model Eq. (2), all the coupling constants (spring constants $k_{1}$ and $k_{2}$ ) are central force in nature. Imagine that one carries out a real-space renormalization-group $(\mathrm{RG})$ calculation on the central-force model Eq. (3), the RG iteration will generate various coupling constants including $\mathrm{NN}$ and NNN coupling constants. Hence we may view Eq. (2) as a renormalized Hamiltonian for the central-force model. Our study of Eq. (2) will provide useful information about the relationship between the central-force model and the bond-bending model and shine 
light on this controversial issue as to whether the centralforce model and the bond-bending model belong to the same universality class.

From Eqs. (3) and (4) one can construct the dynamical matrix and find all the eigenmodes of lattice vibration. A cluster is defined as rigid if there are only three zerofrequency modes in two dimensions (two for translation and one for rotation). Note that Eq. (4) plays the role of the bond-bending term. As a result, a percolation cluster is rigid and we expect that the central-force model with NNN interactions fall in the same universality class of the bondbending model.

In this paper, we have developed a low concentration series expansion up to 18 bonds on a honeycomb lattice. We have calculated the elastic splay crossover exponent $\zeta_{\mathrm{SP}}$ and found that $\zeta_{\mathrm{SP}}=1.29 \pm 0.01$. Recently, we ${ }^{11,15}$ have related the elastic splay crossover exponent $\zeta_{\mathrm{SP}}$ to the bulk modulus exponent $f_{B}$

$$
f_{B}=d \nu+\zeta_{\mathrm{SP}},
$$

where $\nu$ is the correlation length exponent. From Eq. (5), we obtained $f_{B}=3.96 \pm 0.01$ which agrees very well with the most accurate value $f_{B}=3.96 \pm 0.04$ for the bond-bending model obtained by Zabolitzky et al. ${ }^{13}$ using a transfer-matrix technique on the honeycomb lattice. Our result suggests that the central-force model with the NNN interaction and the bond-bending model are in the same universality class.

\section{ELASTIC SUSCEPTIBILITIES AND CROSSOVER EXPONENTS}

To formulate the elastic network, it is useful to recall the nature of the crossover from percolation to conductivity in the analogous randomly diluted resistor network, ${ }^{19,20}$ for which the Hamiltonian, $H$ is

$$
H=\frac{1}{2} \sum_{b} \sigma \epsilon_{b}\left(V_{b 1}-V_{b 2}\right)^{2},
$$

where $V_{b 1}-V_{b 2}$ is the voltage difference across the bond $b$. For this model, the susceptibility $\chi_{\lambda}\left(x, x^{\prime}\right)$ (which plays the role of the correlation function) is taken to be $\mathrm{e}^{12}$

$$
\chi_{\lambda}\left(x, x^{\prime}\right)=\left[\operatorname{Tr}\left\{e^{-H} \Psi_{\lambda}(x) \Psi_{-\lambda}\left(x^{\prime}\right)\right\} / \operatorname{Tr} e^{-H}\right]_{\mathrm{av}},
$$

where $\Psi_{\lambda}(x)=e^{-i \lambda V(x)},[\ldots]_{\mathrm{av}}$ indicates an average over the random bond variables $\epsilon_{b}$, Tr indicates integration over all $V$ variables from $-\infty$ to $+\infty$, and similarly for the displacement in the elastic model. One can write ${ }^{5}$

$$
\chi_{\lambda}\left(x, x^{\prime}\right)=\left[\exp \left(-\frac{1}{2} \lambda^{2} R_{x, x^{\prime}}\right)\right]_{\mathrm{av}},
$$

where $R_{x, x^{\prime}}$ is the resistance between sites $x$ and $x^{\prime}$. We now consider the interpretation of $\chi_{\lambda}$ for large $\sigma$. First of all, note that $R_{x, x^{\prime}}=\infty$ if sites $x$ and $x^{\prime}$ are not in the same cluster, so that

$$
\chi_{\lambda}\left(x, x^{\prime}\right)=\left[\nu_{x, x^{\prime}} \exp \left(-\frac{1}{2} \lambda^{2} R_{x, x^{\prime}}\right)\right]_{\mathrm{av}},
$$

where $\nu_{x, x^{\prime}}$ is the pair-connectedness function of bond percolation: $\nu_{x, x^{\prime}}=1$ if sites $x$ and $x^{\prime}$ are connected (in the same cluster) and is zero otherwise. For large $\sigma$,

$$
\begin{aligned}
\chi_{\lambda}\left(x, x^{\prime}\right) & =\left[\nu_{x, x^{\prime}}\left(1-\frac{\lambda^{2}}{2 \sigma} r_{x, x^{\prime}}+\cdots\right)\right]_{\mathrm{av}} \\
& =\chi^{(p)}\left(x, x^{\prime}\right)\left[1-\frac{\lambda^{2}}{2 \sigma} r_{x, x^{\prime}}^{\mathrm{av}}+\cdots\right] \\
& \sim \chi^{(p)}\left(x, x^{\prime}\right)\left[1-\frac{\lambda^{2}}{2 \sigma}\left|x-x^{\prime}\right| \zeta_{\mathrm{Re}} / \nu\right],
\end{aligned}
$$

where $\chi^{(p)}\left(x, x^{\prime}\right)$ is the susceptibility for percolation and $r_{x, x^{\prime}}^{\mathrm{av}}=\left[\sigma R_{x, x^{\prime}}\right]_{\mathrm{av}} \sim\left|p-p_{c}\right|^{-\zeta_{\mathrm{Re}}}$ is the configurationally averaged dimensionless resistance between sites $x$ and $x^{\prime}$ subject to these sites $x$ and $x^{\prime}$ being in the same cluster. One can say that $\chi_{\lambda}\left(x, x^{\prime}\right)$ defines a percolation problem in the limit $\sigma \rightarrow \infty$. This so-defined percolation problem is, of course, identical to the traditional percolation problem. In this formulation $^{19,20}$ the critical exponents describing the randomly diluted resistor network can be expressed in terms of the exponents for percolation and the crossover exponent $\zeta_{\mathrm{Re}}$ of Eq. (10). Using the node-link picture, ${ }^{21}$ or scaling theory, ${ }^{12,20}$ one obtains

$$
t=(d-2) \nu+\zeta_{\operatorname{Re}},
$$

where $t$ is the conductivity exponent. For the elastic network, the analogy would be to define the elastic susceptibility and the elastic crossover exponent. We define two elastic susceptibilities, $\chi_{\mathrm{SR}}$ and $\chi_{\mathrm{el}}$, where $\chi_{\mathrm{SR}}$ describes the elastic response to the splay distortion and $\chi_{\mathrm{el}}$ the response to the extension. The two-bond splay elastic susceptibility is defined $^{5,16}$ as follows:

$$
\chi_{\mathrm{SR}}\left(b, b^{\prime}\right)=\left[\operatorname{Tr} e^{-H} \Psi_{\lambda}(b) \Psi_{-\lambda}\left(b^{\prime}\right) / \operatorname{Tr} e^{-H}\right]_{\mathrm{av}},
$$

where $\Psi_{\lambda}(b)=\exp \left[i \lambda\left(\overrightarrow{u_{b}} \times \hat{R}_{b}\right) \cdot \hat{k}\right]=\exp \left(i \lambda \theta_{b}\right)$ is the splay order parameter and $\hat{k}$ is an unit vector perpendicular to the two-dimensional plane. Denoting $\chi_{b, b^{\prime}}^{\mathrm{SR}}$ the effective bond angle elastic resistance (i.e., inverse of elastic constant) for the splay distortion defined as the angular displacement of the two bonds divided by the torque needed to maintain the equilibrium, we have $\mathrm{e}^{5,16}$

$$
\chi_{b, b^{\prime}}^{\mathrm{SR}}=\left\langle\theta_{b}-\theta_{b^{\prime}}|G| \theta_{b}-\theta_{b^{\prime}}\right\rangle,
$$

where $G$ is the Green's function defined as $G=\lim _{\epsilon \rightarrow \infty}(V+i \epsilon)^{-1}, V$ is the dynamical matrix. From Eqs. (12) and (13), we obtain

$$
\chi_{\mathrm{SR}}\left(b, b^{\prime}\right)=\left[\exp \left(-\frac{\lambda^{2}}{2 k} \tilde{\chi}_{b, b^{\prime}}^{\mathrm{SR}}\right)\right]_{\mathrm{av}},
$$

where $\tilde{\chi}_{b, b^{\prime}}^{\mathrm{SR}}=k \chi_{b, b^{\prime}}^{\mathrm{SR}}$ and $k \sim k_{1} \sim k_{2}$. Note that $\chi_{b, b^{\prime}}^{\mathrm{SR}}=\infty$ if bonds $b$ and $b^{\prime}$ are not in the same cluster, so that

$$
\chi_{\mathrm{SR}}\left(b, b^{\prime}\right)=\left[\nu_{b, b^{\prime}} \exp \left(-\frac{\lambda^{2}}{2 k} \tilde{\chi}_{b, b^{\prime}}^{\mathrm{SR}}\right)\right]_{\mathrm{av}},
$$

where $\nu_{b, b^{\prime}}$ is the pair-connectedness function. For small $\lambda^{2} / k$, we expand Eq. (15) in powers of $\lambda^{2} / k$ : 


$$
\begin{aligned}
\chi_{\mathrm{SR}}\left(b, b^{\prime}\right) & =\left[\nu_{b, b^{\prime}}\left(1-\frac{\lambda^{2}}{2 k} \tilde{\chi}_{b, b^{\prime}}^{\mathrm{SR}}+\cdots\right)\right]_{\mathrm{av}} \\
& \sim \chi_{p}\left(b, b^{\prime}\right)\left(1-\frac{\lambda^{2}}{2 k}\left|r_{b}-r_{b^{\prime}}\right| \zeta_{\mathrm{SP}} / \nu\right),
\end{aligned}
$$

where $\zeta_{\mathrm{SP}}$ is the elastic splay crossover exponent which describes the way $\left[\chi_{b, b^{\prime}}^{\mathrm{SR}}\right]_{\mathrm{av}}$ scales with the distance and $\chi_{p}\left(b, b^{\prime}\right)$ is the susceptibility for percolation. In the limit $k \rightarrow \infty, \chi_{\mathrm{SR}}\left(b, b^{\prime}\right)$ defines the percolation problem. Therefore, the elastic problem can be described by this crossover exponent $\zeta_{\mathrm{SP}}$

We can also define ${ }^{15,16}$ two-point elastic susceptibility $\chi_{\text {el }}$ similar to Eq. (12):

$$
\begin{aligned}
\chi_{\mathrm{el}}\left(\vec{x}, \overrightarrow{x^{\prime}}\right) & =\left[\operatorname{Tr} e^{-H} \Psi_{\lambda}(\vec{x}) \Psi_{-\lambda}\left(\overrightarrow{x^{\prime}}\right) / \operatorname{Tr} e^{-H}\right]_{\mathrm{av}} \\
& =\left[\exp \left(-\frac{\lambda^{2}}{2 k} \tilde{\chi}_{x, x^{\prime}}^{\mathrm{el}}\right)\right]_{\mathrm{av}},
\end{aligned}
$$

where $\Psi_{\lambda}(\vec{x})=e^{i \lambda \vec{u}_{x} \cdot \hat{R}_{b}}$ and $b$ denotes the bond connecting sites $\vec{x}$ and $\overrightarrow{x^{\prime}}$. Here $\tilde{\chi}_{x, x^{\prime}}^{\mathrm{el}}=k \chi_{x, x^{\prime}}^{\mathrm{el}}$ and

$$
\chi_{x, x^{\prime}}^{\mathrm{el}}=\left\langle\left(\vec{u}_{x}-\vec{u}_{x^{\prime}}\right) \cdot \hat{R}_{b}|G|\left(\vec{u}_{x}-\vec{u}_{x^{\prime}}\right) \cdot \hat{R}_{b}\right\rangle
$$

is the effective two-point elastic resistance between sites $\vec{x}$ and $\overrightarrow{x^{\prime}}$. In the critical region, when $k^{-1}$ is nonzero, the Fourier transform of $\chi_{\mathrm{SR}}\left(b, b^{\prime}\right)$ and $\chi_{\mathrm{el}}\left(\vec{x}, \overrightarrow{x^{\prime}}\right)$ can be written as

$$
\begin{aligned}
\chi_{\mathrm{SR}}(q) & =\left|p-p_{c}\right|^{-\gamma} g_{1}\left(q \xi, k\left|p-p_{c}\right|^{\zeta} \mathrm{SP}\right) \\
& \sim k^{-1}\left|p-p_{c}\right|^{-\gamma-\zeta_{\mathrm{SP}}},
\end{aligned}
$$

and

$$
\chi_{\mathrm{el}}(q)=\left|p-p_{c}\right|^{-\gamma} g_{2}\left(q \xi, k\left|p-p_{c}\right|^{\zeta \mathrm{el})} \sim k^{-1}\left|p-p_{c}\right|^{-\gamma-\zeta_{\mathrm{el}}},\right.
$$

where $g_{1}$ and $g_{2}$ are scaling functions. Here we have introduced another crossover exponent $\zeta_{\mathrm{el}}$ describing the way the two-point elastic constant scales with the distance.

We now use a scaling argument ${ }^{11,15,16}$ to relate $\zeta_{\text {SP }}$ and $\zeta_{\text {el }}$ to $f_{B}$, the elastic bulk modulus exponent. For the resistor network, one has the spatial Fourier transform of the voltagevoltage correlation function [denoted $\langle V(q) V(0)\rangle \sim \chi_{\lambda}(q)$ ] at $p<p_{c}$ :

$$
\langle V(q) V(0)\rangle=\left|p-p_{c}\right|^{\gamma} f\left(q \xi, \sigma\left|p-p_{c}\right|^{\zeta \mathrm{Re}}\right),
$$

where $f$ is some scaling function. For $p>p_{c}$ we have $\mathrm{e}^{12}$

$$
\langle V(q) V(0)\rangle=\frac{P(p)^{2}}{\Sigma(p) q^{2}},
$$

where $P(p)$ is the fraction of sites in the infinite cluster and $\Sigma(p)$ is the bulk conductivity. We assume that the form of Eq. (20) also holds for $p>p_{c}$, in which case it assumes the form written explicitly in Eq. (21). Then it follows that

$$
\Sigma(p)=\frac{P(p)^{2}\left|p-p_{c}\right|^{\gamma}}{q^{2} f\left(q \xi, \sigma\left|p-p_{c}\right|^{\left.\zeta_{\mathrm{Re}}\right)}\right.} .
$$

Since $\Sigma(p)$ is proportional to $\sigma$, we then have

$$
\begin{aligned}
\Sigma(p) & \sim \sigma \xi^{2}\left|p-p_{c}\right|^{\gamma+2 \beta+\zeta_{\mathrm{Re}}} \\
& \sim \sigma\left|p-p_{c}\right|^{\gamma+2 \beta+\zeta_{\mathrm{Re}}-2 \nu} \sim \sigma\left|p-p_{c}\right|^{t},
\end{aligned}
$$

and using the scaling relation $\gamma+2 \beta=2-\alpha$ and the hyperscaling relation $d \nu=2-\alpha$ we obtain Eq. (11).

For the elastic network, we consider the angle-angle correlation function ${ }^{11,15,16}$

$$
\begin{aligned}
\langle\delta \theta \delta \theta\rangle & =\langle|\nabla \times \vec{u}||\nabla \times \vec{u}|\rangle \\
& =\frac{P(p)^{2}}{\mu(p)} \sim\left|p-p_{c}\right|^{-\gamma} g_{1}\left(q \xi, k\left|p-p_{c}\right|^{\left.\zeta_{\mathrm{SP}}\right)},\right.
\end{aligned}
$$

where $g_{1}$ is the scaling function defined in Eq. (18) and $\mu(p)$ is the shear modulus. We get

$$
\mu(p)=\frac{P(p)^{2}\left|p-p_{c}\right|^{\gamma}}{g_{1}\left(q \xi, k\left|p-p_{c}\right|^{\left.\zeta_{\mathrm{SP}}\right)}\right.} \sim k\left|p-p_{c}\right|^{\gamma+2 \beta+\zeta_{\mathrm{SP}}} .
$$

By assuming $B$ and $\mu$ to have the same critical behavior, ${ }^{13}$ we have

$$
f_{B}=d \nu+\zeta_{\mathrm{SP}}
$$

Similarly if we consider the $\langle u u\rangle$ correlation function, we obtain another relation ${ }^{11,16}$

$$
f_{B}=(d-2) \nu+\zeta_{\mathrm{el}} .
$$

From Eqs. (26) and (27), we have

$$
\zeta_{\mathrm{el}}=2 \nu+\zeta_{\mathrm{SP}}
$$

Equation (27) can also be obtained using the node-link picture or node-link and blob picture. ${ }^{21,2,22}$ Near the percolation threshold $p_{c}$, the percolation backbone can be described by the node-link and blob picture, ${ }^{21,22}$ where the system is made up by long one-dimensional strings or links with blobs connected at nodes. Typical separation between the nodes is $\xi$ which is the correlation length for percolation. According to our definition the effective elastic constant for a string is $1 / \chi_{x, x^{\prime}}^{\text {el }}$. From the node-link and blob picture ${ }^{21,2}$ the bulk modulus $B$ scales like $\xi^{2-d} / \chi_{x, x^{\prime}}^{\mathrm{el}}$, from which we arrive at Eq. (27).

\section{SERIES EXPANSION AND ANALYSIS}

To calculate the exponent $\zeta_{\mathrm{SP}}$ and $\zeta_{\mathrm{el}}$, we have developed series expansions in powers of $p$ up to $p^{18}$ on the honeycomb lattice for the following quantities:

$$
\begin{aligned}
& \chi_{\mathrm{SR}}=\left[\sum_{b} \chi_{b, b^{\prime}}^{\mathrm{SR}}\right]_{\mathrm{av}} \equiv \sum_{\Gamma} P(\Gamma) \sum_{b \in \Gamma} \chi_{b, b^{\prime}}^{\mathrm{SR}} \\
& \equiv \sum_{n} a_{n} p^{n} \sim\left|p-p_{c}\right|^{-\gamma-\zeta_{\mathrm{SP}}} \\
& \chi_{\mathrm{el}}=\left[\sum_{x} \chi_{x, x^{\prime}}^{\mathrm{el}}\right]_{\mathrm{av}} \equiv \sum_{\Gamma} P(\Gamma) \sum_{x \in \Gamma} \chi_{x, x^{\prime}}^{\mathrm{el}} \\
& \equiv \sum_{n} b_{n} p^{n} \sim\left|p-p_{c}\right|^{-\gamma-\zeta_{\mathrm{el}}},
\end{aligned}
$$


TABLE I. Series coefficients $a_{n}$ and $b_{n}$.

\begin{tabular}{rrr}
\hline \hline$n$ & \multicolumn{1}{c}{$a_{n}$} & \multicolumn{1}{c}{$b_{n}$} \\
\hline 1 & 3.0000 & 0.0000 \\
2 & 6.0000 & 60.0000 \\
3 & 77.7143 & 208.0000 \\
4 & 401.5714 & 565.0000 \\
5 & 1421.6669 & 1467.6667 \\
6 & 2426.7332 & 2368.8000 \\
7 & 8302.4782 & 6505.4867 \\
8 & 21261.5948 & 12879.7160 \\
9 & 52696.9183 & 26476.3224 \\
10 & 91294.4947 & 38488.9855 \\
11 & 271399.5287 & 108945.1960 \\
12 & 499114.6696 & 157841.5476 \\
13 & 1131285.9690 & 316181.8901 \\
14 & 2103714.2723 & 520638.8864 \\
15 & 5141108.5374 & 1216143.2404 \\
16 & 8112170.7968 & 1424601.4426 \\
17 & 20063145.7472 & 3750446.1292 \\
18 & 31912709.6575 & 4761271.8424 \\
\hline \hline
\end{tabular}

where $\chi_{b, b^{\prime}}^{\mathrm{SR}}$ and $\chi_{x, x^{\prime}}^{\mathrm{el}}$ have been defined in Sec. II, the summation $\Sigma_{\Gamma}$ is over all clusters, $P(\Gamma)$ is the associated probability per site that the cluster $\Gamma$ occurs. For the resistor network, two clusters which are topologically equivalent give the same two-point resistance. For the elastic network, however, clusters with different shapes give different values of elastic response. In calculating the series for $\chi_{\mathrm{SR}}$ and $\chi_{\mathrm{el}}$ we have to count every cluster with different shape, which is very time consuming.

The series up to order $p^{18}$ on the honeycomb lattice are presented in Table I. For the model that we considered, because of the NNN interaction, the rigidity percolation threshold is the same as that for the usual bond percolation on the honeycomb lattice, $p_{c}=0.6527$, so it is much easier to analyze the series.

We have analyzed the series with two different methods, ${ }^{23}$ one based on the assumption that there are nonanalytic confluent corrections to scaling and another ${ }^{24}$ based on the assumption that there are logarithmic confluent corrections. Nonanalytic confluent corrections to scaling ${ }^{13}$ have several origins, including irrelevant operators. They are definitely present in both isotropic and directed two-dimensional percolation and thus must be allowed for in the series discussed in this paper. There is also evidence from simulation by Zabolitzky et al. ${ }^{13}$ that there may be logarithmic corrections to elastic critical behavior in two dimensions.

To analyze the series, denoted by $\chi(p)$ in general, we assume that the series has the form

$\chi(p) \sim a\left(p_{c}-p\right)^{-h}\left[1+b\left(p_{c}-p\right)^{\Delta_{1}}+\ldots\right], \quad p<p_{c}$,

where $h$ is the critical exponent that we wish to determine, and $p_{c}$ is the critical point. We first transform the series in $p$ into the series in the variable $y$, where

$$
y=1-\left(1-p / p_{c}\right)^{\Delta_{1}},
$$

and then take Padé approximants ${ }^{25}$ to

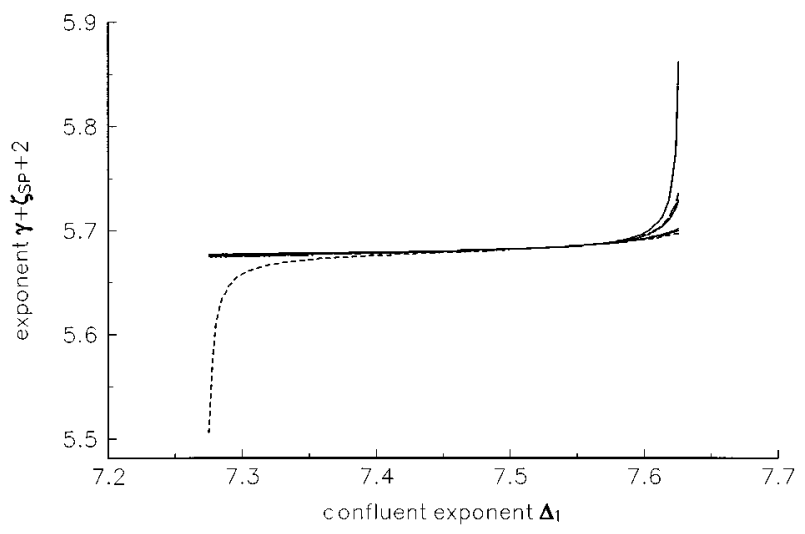

FIG. 1. Graph of Padé approximants to $\gamma+\zeta_{\mathrm{SP}}+2$ as a function of $\Delta_{1}$ at $p_{c}$ for the $\chi_{\mathrm{SR}}^{\prime \prime}$ series.

$$
G(y)=\Delta_{1}(y-1) \frac{d}{d y} \ln (\chi),
$$

which should converge to $-h$. Since we already know the critical point $p_{c}$ we can simply plot graphs of $h$ versus the input $\Delta_{1}$ and choose $\Delta_{1}$ such that all Padé approximants give as closely as possible the same values of $h$.

The assumption of logarithmic corrections entails fitting to the form

$$
\chi(p) \sim\left(p_{c}-p\right)^{-h}\left|\ln \left(p_{c}-p\right)\right|^{\theta}, \quad p<p_{c} .
$$

We fitted this form with the method of Adler and Privman. ${ }^{24}$ The analysis of the logarithmic form involves taking Padé approximants to the series

$$
H(p)=-\left(p_{c}-p\right)\left|\ln \left(p_{c}-p\right)\right|\left\{\left(\chi^{\prime} / \chi\right)-\left[h /\left(p_{c}-p\right)\right]\right\},
$$

so that $H(p)$ goes to $\theta$ as $p \rightarrow p_{c}$. To get the exponent $h$, we take Padé approximants to $H(p)$ at $p_{c}$ to obtain graphs of $\theta$ as a function of $h$.

From our previous experience, ${ }^{16,17}$ the convergence of the $\chi_{\mathrm{SR}}$ series is better than $\chi_{\mathrm{el}}$. A graph of Padé approximants from $\chi_{\mathrm{SR}}^{\prime \prime}$ series is given in Fig. 1 assuming the scaling form given in Eq. (31), where $\chi_{\mathrm{SR}}^{\prime \prime}$ is the second derivative of the series $\chi_{\mathrm{SR}}$. All curves converge around $\Delta_{1}=7.50$, from which we estimate $h=\gamma+\zeta_{\mathrm{SP}}+2=5.68 \pm 0.01$ or $\zeta_{\mathrm{SP}}=1.29 \pm 0.01$, where we have used the exact value $\gamma=2.3888 \ldots{ }^{26}$ and the error bar is somewhat subjective. We have also tested the logarithmic corrections for the series $\chi_{\mathrm{SR}}$ and found no convergence. For the series $\chi_{\text {el }}$ we have fitted the series $\chi_{\mathrm{el}}^{\prime}$ to the form of Eq. (32). This is because there is indication ${ }^{13}$ that the logarithmic corrections exist in the bond-bending model and from our previous series analysis for the bond-bending model we found the logarithmic corrections in the series $\chi_{\mathrm{el}}$. We found that $h=\gamma+\zeta_{\mathrm{el}}+1=7.39 \pm 0.09$ and $\theta=-0.5$. This gives $\zeta_{\mathrm{el}}=4.00 \pm 0.09$ which satisfys the relation Eq. (28). The reason that the series $\chi_{\mathrm{el}}$ gives a poor result is the following: in calculating the series $\chi_{\mathrm{el}}$, we calculate the effective twopoint elastic resistance $\chi_{x, x^{\prime}}^{\mathrm{el}} \sim u_{x, x^{\prime}} / F_{x, x^{\prime}}$ between sites $x$ and $x^{\prime}$, where $u_{x, x^{\prime}}$ is the displacement along $\hat{r}_{x, x^{\prime}}$, the unit vector connecting sites $x$ and $x^{\prime}$ and $F_{x, x^{\prime}}$ is the force along 
$\hat{r}_{x, x^{\prime}}$ needed to maintain the equilibrium. Hence $\chi_{x, x^{\prime}}^{\mathrm{el}}$ depends on $\hat{r}_{x, x^{\prime}}$ which will affect the result for small systems or small clusters. Using $\zeta_{\mathrm{SP}}=1.29 \pm 0.01$ and Eq. (26), we obtain $f_{B}=3.96 \pm 0.01$, which is the same as the bulk modulus exponent for the bond-bending model. This shows that the central-force model with the NNN interaction and the bond-bending model are in the same universality class.

In summary, we have calculated the elastic crossover exponents, $\zeta_{\mathrm{SP}}$ and $\zeta_{\mathrm{el}}$, for the central-force model with the NNN interaction using the series-expansion method on the honeycomb lattice. We found that $\zeta_{\mathrm{SP}}=1.29 \pm 0.01$ and $\zeta_{\mathrm{el}}=4.00 \pm 0.09$. From the scaling theory $f_{B}=d \nu+\zeta_{\mathrm{SP}}$, we obtain $f_{B}=3.96 \pm 0.01$ which is very close to the bulk modulus exponent for the bond-bending model obtained by Zabolitzky et al. This suggests that the central-force model with the NNN interaction and the bond-bending model are in the same universality class. When enforcing the logarithmic correction, the exponent $\zeta_{\mathrm{el}}$ is consistent with the scaling theory $\zeta_{\mathrm{el}}=2 \nu+\zeta_{\mathrm{SP}}$.

\section{ACKNOWLEDGMENTS}

I would like to thank Professor A. B. Harris for helpful discussion. This work is supported by a CRCG grant at The University of Hong Kong.
${ }^{1}$ S. Feng and P. N. Sen, Phys. Rev. Lett. 52, 216 (1984).

${ }^{2}$ Y. Kantor and I. Webman, Phys. Rev. Lett. 52, 1891 (1984).

${ }^{3}$ M. A. Lemieux, P. Breton, and A. M. S. Tremblay, J. Phys. (Paris) Lett. 46, L1 (1985); A. R. Day, R. R. Tremblay, and A. M. S. Tremblay, Phys. Rev. Lett. 56, 2501 (1986).

${ }^{4}$ S. Feng, P. N. Sen, B. I. Halperin, and C. J. Lobb, Phys. Rev. B 30, 5386 (1984).

${ }^{5}$ J. Wang and A. B. Harris, Phys. Rev. Lett. 55, 2459 (1985).

${ }^{6}$ S. Roux and A. Hansen, Europhys. Lett. 6, 301 (1988).

${ }^{7}$ S. Feng, Phys. Rev. B 32, 510 (1985).

${ }^{8}$ J. Wang, J. Phys. A 22, L401 (1989).

${ }^{9}$ M. Sahimi, J. Phys. C 19, L79 (1986).

${ }^{10}$ S. Roux, J. Phys. A 19, L351 (1986).

${ }^{11}$ A. B. Harris and T. C. Lubensky (unpublished).

${ }^{12}$ A. B. Harris and R. Fisch, Phys. Rev. Lett. 38, 796 (1977); R. Fisch and A. B. Harris, Phys. Rev. B 18, 416 (1978).

${ }^{13}$ J. G. Zabolitzky, D. J. Bergman, and D. Stauffer, J. Stat. Phys. 44, 211 (1986).

${ }^{14}$ J. G. Zabolitzky, Phys. Rev. B 30, 4077 (1985); C. J. Lobb and D. J. Frank, ibid. 30, 4090 (1984); H. J. Herrnamm, B. Derrida, and
J. Vannimenus, ibid. 30, 4090 (1984); D. C. Hong, S. Havlin, H.

J. Herrmann, and H. E. Stanley, ibid. 30, 4083 (1984); all give $\zeta_{\mathrm{Re}} \approx 1.30$ in two dimensions.

${ }^{15}$ J. Wang and A. B. Harris, Europhys. Lett. 6, 615 (1988).

${ }^{16}$ J. Wang, A. B. Harris, and J. Adler, Phys. Rev. B 45, 7084 (1992).

${ }^{17}$ J. Wang, J. Phys. A, 28, L415 (1995).

${ }^{18}$ E. J. Garboczi and M. F. Thorpe, Phys. Rev. B 31, 7276 (1985).

${ }^{19}$ M. J. Stephen, Phys. Rev. B 17, 4444 (1978).

${ }^{20}$ A. B. Harris and T. C. Lubensky, Phys. Rev. B 35, 6964 (1987).

${ }^{21}$ A. S. Skal and B. I. Shklovskii, Fiz. Tekh. Poluprovodn. 8, 1582 (1974) [Sov. Phys. Semicond. 8, 1029 (1975)]; P. G. de Gennes, J. Phys. (Paris) Lett. 37, L1 (1976).

${ }^{22}$ A. Coniglio, J. Phys. A 15, 3829 (1982).

${ }^{23}$ J. Adler, M. Moshe, and V. Privman, Phys. Rev. B 26, 1411 (1982); J. Phys. A 14, L363 (1981).

${ }^{24}$ J. Adler and V. Privman, J. Phys. A 14, L463 (1981).

${ }^{25}$ J. H. Chen and M. E. Fisher, J. Phys. A 14, 2553 (1981).

${ }^{26}$ M. P. M. den Nijs, J. Phys. A 12, 1857 (1979); M. P. Nightingale and H. W. J. Blote, Physica 104A, 352 (1980). 